\title{
Soporte social percibido y competencia para el cuidado en el hogar, Ocaña, Colombia ${ }^{1}$
}

Social Support Perceived and Competence for Care in the Home, Ocaña, Colombia

Olga Marina Vega Angarita², Dianne Sofía González Escobar ${ }^{3}$

\section{Resumen}

Objetivo. Describir y determinar la relación entre el nivel de percepción de soporte social y la competencia para cuidar en el hogar de cuidadores familiares de personas adultas con enfermedad crónica. Método. Estudio correlacional con abordaje cuantitativo y de corte transversal realizado en una muestra de 103 cuidadores del municipio de Ocańa. Se utilizaron tres instrumentos: Ficha de caracterización Cuidador - persona con enfermedad crónica GCPC-UN-C, Soporte Social en enfermedad crónica de Hilbert (SSCI) y Competencia para el cuidado - Cuidador versión abreviada- GCPC-UN-CPC. Resultados. Respecto al soporte social se encontró satisfacción en todas las dimensiones de la escala de Hilbert y a nivel global. El nivel de competencia global para el cuidado en el hogar de los cuidadores fue adecuado; sin embargo se identifican necesidades de fortalecimiento en el conocimiento, lo instrumental y unicidad. Se identificó una correlación débil con baja significancia estadística entre las variables a estudio. Conclusiones. La competencia para cuidar en el hogar en el cuidador se desarrolla de manera independiente del nivel de satisfacción con el soporte social percibido.

Palabras claves: Cuidadores, Enfermedad crónica, Competencia, Soporte social, Adultos, Cuidado de enfermería (DeCS).

\footnotetext{
1. Corresponde al resultado parcial del estudio denominado "Apoyo social y competencia para el cuidado de cuidadores familiares de personas en situación de enfermedad crónica del Norte de Santander", realizado en el marco de un proyecto conjunto del Grupo de Cuidado de Enfermería de la Universidad Francisco de Paula Santander, con la Red Latinoamericana de Cuidado del Enfermo Crónico y su familia (Cronifam)

2. Universidad Francisco de Paula Santander. Av Gran Colombia \# 12 E - 96 Barrio Colsag. Cúcuta, Colombia. Enfermera. Doctora en

Educación.

3. Universidad Francisco de Paula Santander. Av Gran Colombia \# 12 E - 96 Barrio Colsag. Cúcuta, Colombia. Enfermera. Magister en Enfermería.
} 


\section{Abstract}

Objective. Describe and determine the relationship between the level of perception of social support and the competence to care at home for family caregivers of adults with chronic disease. Method. Correlational study with a quantitative and cross-sectional approach performed on a sample of 103 caregivers from the municipality of Ocańa. Three instruments were used: Characterization card Caregiver - person with chronic disease GCPC-UN-C, Social Support in chronic Hilbert disease (SSCI) and Competence for care - Caregiver abridged version- GCPC-UN-CPC. Results. Regarding social support, satisfaction was found in all dimensions of the Hilbert scale and globally. The level of global competence for caregiving at home was adequate. However, needs for strengthening knowledge, instrumentality and uniqueness are identified. We identified a weak correlation with low statistical significance among the variables under study. Conclusions. The competence to care at home in the caregiver is developed independently of the level of satisfaction with the perceived social support.

Keywords: Caregivers, Chronic illness, Competence, Social support, Adults, Nursing care (DeCS).

\section{Introducción}

\section{Situación del envejecimiento y de las enfermedades no transmisibles}

El envejecimiento de la población es un fenómeno que se está produciendo en el mundo. Varias razones explican este hecho: a) avances de la tecnología y del conocimiento científico de la última mitad del siglo XX han permitido mayor expectativa de vida de la poblacion; b) disminución de la mortalidad; c) control de las enfermedades infecciosas y parasitarias y, d) mejoramiento de las condiciones sanitarias (1).

Se calcula que hay 962 millones de personas con 60 ańos o más, es decir, un 13 por ciento de la población mundial (2). La nación y región están en esa línea; de los 48 millones de colombianos, aproximadamente $10 \%$ (es decir, 5,2 millones) son mayores de 60 ańos (3).

Cambios evidentes en la conformación del patrón de mortalidad actual por enfermedades no transmisibles asignables en esta poblacion son uno de los temas de mayor importancia para los sistemas de salud y de protección social, que deben ofrecer atención a un gran contingente de personas mayores. Repercusiones económicas generadas por los gastos de atención profesional requeridos en la prevención, diagnóstico, tratamiento y rehabilitación de la enfermedad han planteado nuevas formas de atención y el cuidado de los enfermos crónicos se convierte en un aspecto más de la vida familiar. 
En el entorno colombiano es frecuente que la familia asuma el cuidado de sus familiares longevos con un peso cuantitativo muy importante. Según datos del 2010, uno de cada tres hogares cuenta con la presencia de una persona mayor, y lo que es aún más significativo, uno de cada cuatro hogares es liderado por personas mayores (4).

Se han observado de manera repetida rasgos sociodemográficos comunes en los cuidadores. Fuertes nexos de género (mujeres), y parentesco (esposa, hija, madre); quienes comparten múltiples actividades cotidianas, sean estas básicas, instrumentales o avanzadas (5), sobrepasados en dedicación y exigencia de cuidado prolongado; con escaza preparación e inadecuada competencia (67), y baja percepción de soporte social (8).

El soporte social es un aspecto inherente a los cuidados que se intercambian en las familias, tanto en situaciones cotidianas como de crisis (9); se constituye en fuente natural de apoyo y de ayuda disponible para los cuidadores de enfermos crónicos. El presente estudio adopta el concepto de soporte social dado por Hilbert (10) entendido como la diversidad de comportamientos naturales de ayuda a los individuos y son receptores en interacciones sociales. Se compone de cinco dimensiones: interacción personal, guía, información/instrucción, ayuda tangible e interacción social.

El conocimiento, las habilidades y destrezas, las actitudes y disposición han sido referidos como elementos claves de un des- empeño competente de los cuidadores. Para Carrillo y colaboradores (8), la competencia supone la capacidad, habilidad y preparación que tiene la persona con enfermedad crónica o su cuidador familiar para ejercer la labor de cuidar en su sitio de vivienda. Se asocian a factores de tipo personal y del contexto propio donde se genera el cuidado (9). Se representan en seis categorías que son: conocimiento, unicidad o condiciones particulares, instrumental procedimental, disfrute de condiciones mínimas para el cuidado o nivel de bienestar, anticipación, y relación social e interacción.

Un aspecto fundamental en la calidad de vida del enfermo crónico es el correspondiente a la percepción adecuada del soporte social y cualificación de los cuidadores. El objetivo del estudio fue describir y determinar la relación entre el nivel de percepción de soporte social y la competencia para cuidar en el hogar de cuidadores familiares de personas adultas con enfermedad crónica.

\section{Materiales y métodos}

Se llevó a cabo un estudio correlacional con abordaje cuantitativo y de corte transversal entre los ańos 2017 y 2018 con el objetivo de determinar la relación entre la percepción del soporte social y la competencia para cuidar en el hogar. Se tomó como poblacion cuidadores residentes en la provincia de Ocaña. La obtención del tamaño muestral se realizó mediante la fórmula para la estimación de una proporción poblacional, con un nivel de confianza del 95\%, una preva- 
lencia esperada del 50\% y un error máximo del $5 \%$. Se obtuvo una muestra final de 103 participantes. Los criterios para la selección fueron: ser cuidador de un adulto mayor con enfermedad crónica, con tiempo mínimo de 6 meses y tener 18 ańos o más años. Se excluyeron cuidadores con afectación cognitiva y conductual.

El estudio se rigió por lo establecido en la normatividad ética en la investigación en Colombia, Resolución 008430 de 1993 (11) y principios éticos de la declaración de Helsinki. Fueron consideradas las normas actuales de propiedad intelectual y contó con el aval institucional y consentimiento informado de los participantes previa explicación del objetivo del estudio, procedimiento y grado de participación.

En la recolección de la información fueron empleados 3 instrumentos desarrollados y validados en el contexto latinoamericano por el grupo de cuidado al enfermo crónico de la Universidad Nacional de Colombia quienes autorizaron su aplicación: Ficha de caracterización de la Diada Cuidador - persona con enfermedad crónica GCPC-UN-D, que contiene 15 ítems en tres dimensiones: perfil socio demográfico, percepción de carga, apoyo y uso de las tecnologías de información y comunicación. Instrumento Soporte Social en enfermedad crónica de Hilbert (SSCI), que consta de 34 preguntas agrupadas en cinco sub-escalas: interacción personal, guía, información/instrucción, retroalimentación, ayuda tangible e interacción social; con escala tipo
Likert de 1 a 6 , con una puntuación máxima es de 228, a mayor puntaje, mayor percepción de apoyo social. La competencia para el cuidado - Cuidador versión abreviadaGCPC-UN-CPC, se compone de 20 ítems que se miden con una escala tipo Likert de puntuaciones de 0 a 3; distribuidas en seis categorías ordenadas bajo el acróstico CUIDAR: Conocimiento, Unicidad o condiciones particulares, Instrumental procedimental, Disfrute de condiciones mínimas para el cuidado o nivel de bienestar, Anticipación, y Relación social e interacción (12).

En el análisis descriptivo de los datos fueron utilizadas distribuciones de frecuencia simple, cálculo de medidas descriptivas para variables numéricas. La asociación se evaluó con la prueba de Chi Cuadrado, bajo un nivel de significancia de 0.05. En el análisis de correlación entre escalas se utilizó el coeficiente Rho de Spearman. Para la comparación entre dos grupos se utilizó la prueba U de Mann Whitney, mientras para comparación entre más de dos grupos se utilizó la prueba H de Kruskal Wallis.

\section{Resultados}

\section{Variables Sociodemográficas}

La Tabla 1 describe variables sociodemográficas de los cuidadores $(n=103)$. Se reportó una edad promedio de $32.9 \pm 12.6$ años, en un rango entre 18 y 68 ańos; principalmente mujeres (59.2\%), aunque se encontró un porcentaje significativo $(40.8 \%)$ de cuidadores hombres. Predominó la secundaria 
como nivel de escolaridad (41.7\%). En cuanto al estado civil el $53.4 \%$ son personas solteras, en su mayoría dedicada a las labores del hogar (39.9\%); aunque el $35.9 \%$ de los cuidadores se desempeña como trabajadores independientes. La totalidad de los participantes se ubicaron en estratos socioeconómico bajo (1 y 2).

Cada cuidador lleva desempeñando su rol en un promedio de $4.7 \pm 4.9$ años, aunque el $13 \%$ de los cuidadores lleva entre 10 y 30 años dedicado a esta labor con su familiar paciente. El 68.9\% de los cuidadores cuidan a la persona a su cargo desde el momento del diagnóstico, con parentesco hijos(as) y nietos $(54.4 \%$ y $15.5 \%$ respectivamente). Respecto al número de horas diarias que se requieren para el cuidado; el promedio oscila entre 12 y 13 horas al día, aunque hay pacientes que requieren de la presencia del cuidador las 24 horas

Tabla 1. Variables sociodemográficas del cuidador familiar, Provincia de Ocaña. 2017-2018 (n = 103).

\begin{tabular}{|c|c|}
\hline Características sociodemográficas & $\begin{array}{c}\text { Cuidador } \\
\mathrm{n}=113\end{array}$ \\
\hline \multicolumn{2}{|l|}{ Edad (años) } \\
\hline Promedio (ds) & $32,9(12,6)$ \\
\hline Rango & $18-68$ \\
\hline \multicolumn{2}{|l|}{ Sexo: } \\
\hline Femenino & 59,2 \\
\hline Masculino & 40,8 \\
\hline \multicolumn{2}{|l|}{ Grado máximo de escolaridad (\%) } \\
\hline Ninguna & 4,9 \\
\hline Primaria & 30,1 \\
\hline Secundaria & 41,7 \\
\hline Técnico & 11,7 \\
\hline Profesional & 10,7 \\
\hline \multicolumn{2}{|l|}{ Estado civil (\%) } \\
\hline Casado(a) & 14,6 \\
\hline Unión libre & 29,1 \\
\hline Viudo(a) & 1 \\
\hline Soltero(a) & 53,4 \\
\hline Separado(a) & 1,9 \\
\hline \multicolumn{2}{|l|}{ Ocupación (\%) } \\
\hline Hogar & 39,9 \\
\hline Independiente & 35,9 \\
\hline Otras & 24,2 \\
\hline \multicolumn{2}{|l|}{ Nivel socioeconómico (\%) } \\
\hline Estrato 1 & 93,2 \\
\hline Estrato 2 & 6,8 \\
\hline
\end{tabular}

Fuente. análisis de resultados aplicación instrumento SSCI. 
La Tabla 2 describe la percepción nivel de Se evidenció asociación significativa entre percepción de carga según escala de Zarit. la percepción de carga de los pacientes y el Los cuidadores $(\mathrm{n}=103)$ mostraron sobre- nivel de sobrecarga de los cuidadores $(\mathrm{p}<$ carga intensa en el $16.5 \%$, sobrecarga leve 0.01 ).

en el $78.6 \%$ y no sobrecarga en el $4.9 \%$.

Tabla 2. Percepción del nivel de sobrecarga del cuidador familiar, Provincia de Ocańa. 2017-2018 ( $\mathrm{n}=103)$.

\begin{tabular}{|c|c|c|}
\hline \multicolumn{3}{|c|}{ NIVEL DE PERCEPCIÓN DE CARGA SEGÚN ESCALA ZARIT } \\
\hline No Sobrecarga & Sobrecarga Leve & Sobrecarga Intensa \\
\hline $4.9 \%(5)$ & $78,6 \%(81)$ & $16,5 \%(17)$ \\
\hline
\end{tabular}

Fuente. Instrumento percepción de carga según escala de Zarit.

\section{Soporte social percibido en enfermedad crónica}

En la Figura 1 se presenta el resultado del soporte social percibido en los cuidadores $(\mathrm{n}=103)$. A nivel general se observó un pro- medio de $209.8 \pm 24.9$ puntos. Las puntuaciones oscilaron entre 110 y 228 puntos (ver Figura 2). Aunque se observaron valores atípicos, a nivel general, el apoyo percibido por los cuidadores fue satisfactorio a nivel global y en los 5 subescalas.

Figura 1. Variable Soporte social percibido en enfermedad crónica del cuidador familiar, Provincia de Ocaña. 2017-2018 $(\mathrm{n}=103)$.

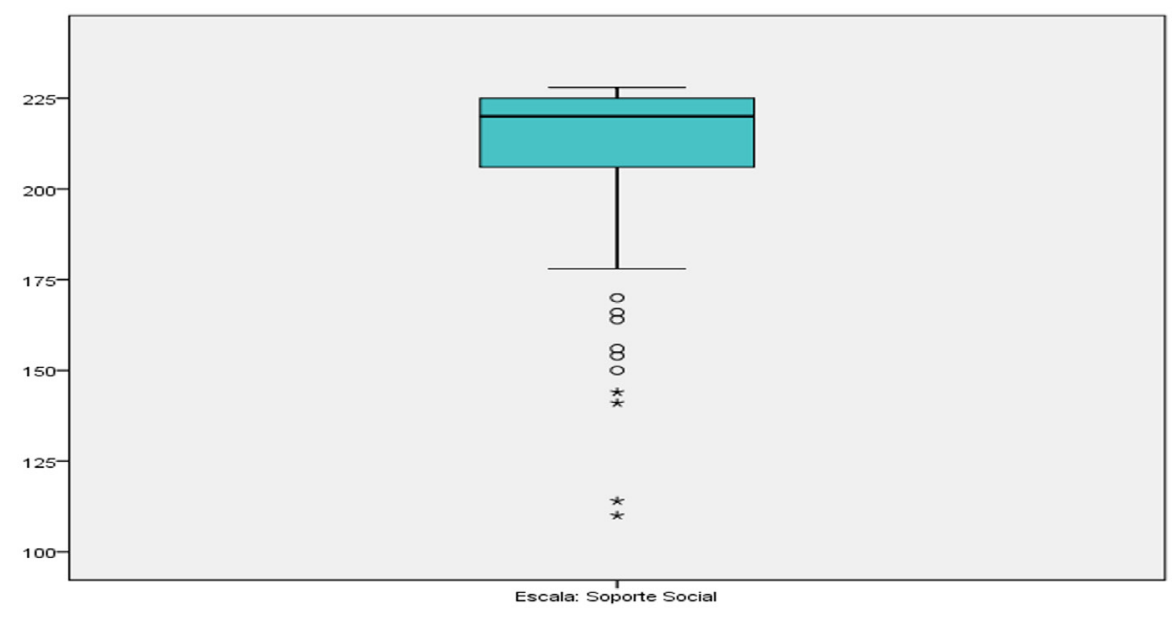

Fuente. Análisis resultados aplicación instrumento GCPC-UN-C.

Competencia para el cuidado en el hogar

A nivel general, la puntuación media de la escala CUIDAR fue de 55.2 \pm 4.9 , con ran- go entre 40 y 60 puntos. En la clasificación de las puntuaciones por tipo de competencia, se pudo establecer que a nivel general el grado de competencia para el cuidado es alto en la mayoría de los cuidadores (89.3\%). 
Con la misma tendencia se reportan altos les medios en las categorías conocimiento, niveles de competencia en la totalidad de unicidad e instrumental, competencias que dimensiones que valora el instrumento ( $\mathrm{Fi}$ - ameritan ser fortalecidas en estos cuidadogura 2). A pesar de ello, se registran nive- res.

Figura 2. Clasificación del nivel de competencia global para el cuidado en el hogar, Provincia de Ocańa. 2017 - 2018 $(\mathrm{n}=103)$.

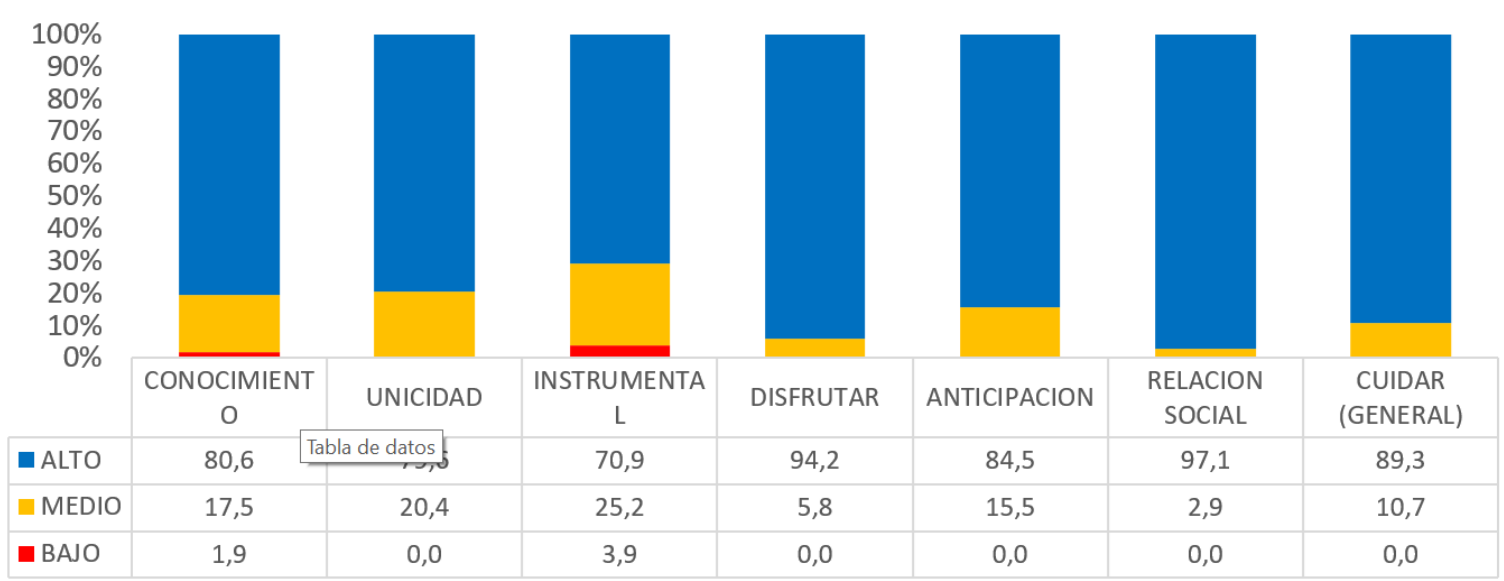

Fuente. Instrumento Competencia para el cuidado - Cuidador versión abreviada- GCPC-UN-CPC.

Correlación entre soporte social percibido en enfermedad crónica y competencia para el cuidado en el hogar

En la Tabla 3 se muestran la matriz de correlación obtenida entre el soporte social y competencia para el cuidado en el hogar. Se logró determinar que no existe correlación entre las puntuaciones globales $(r=-0.03)$, ni tampoco entre sus escalas o dimensiones, pero sí al interior de las dimensiones de cada escala. Lo anterior permite concluir que el comportamiento de la competencia para el cuidado se manifiesta de manera independiente al soporte social brindado a estos cuidadores. 
Tabla 3. Matriz de correlación entre las puntuaciones de competencia para el cuidado y soporte social, Provincia de Ocańa. 2017- $2018(\mathrm{n}=103)$.

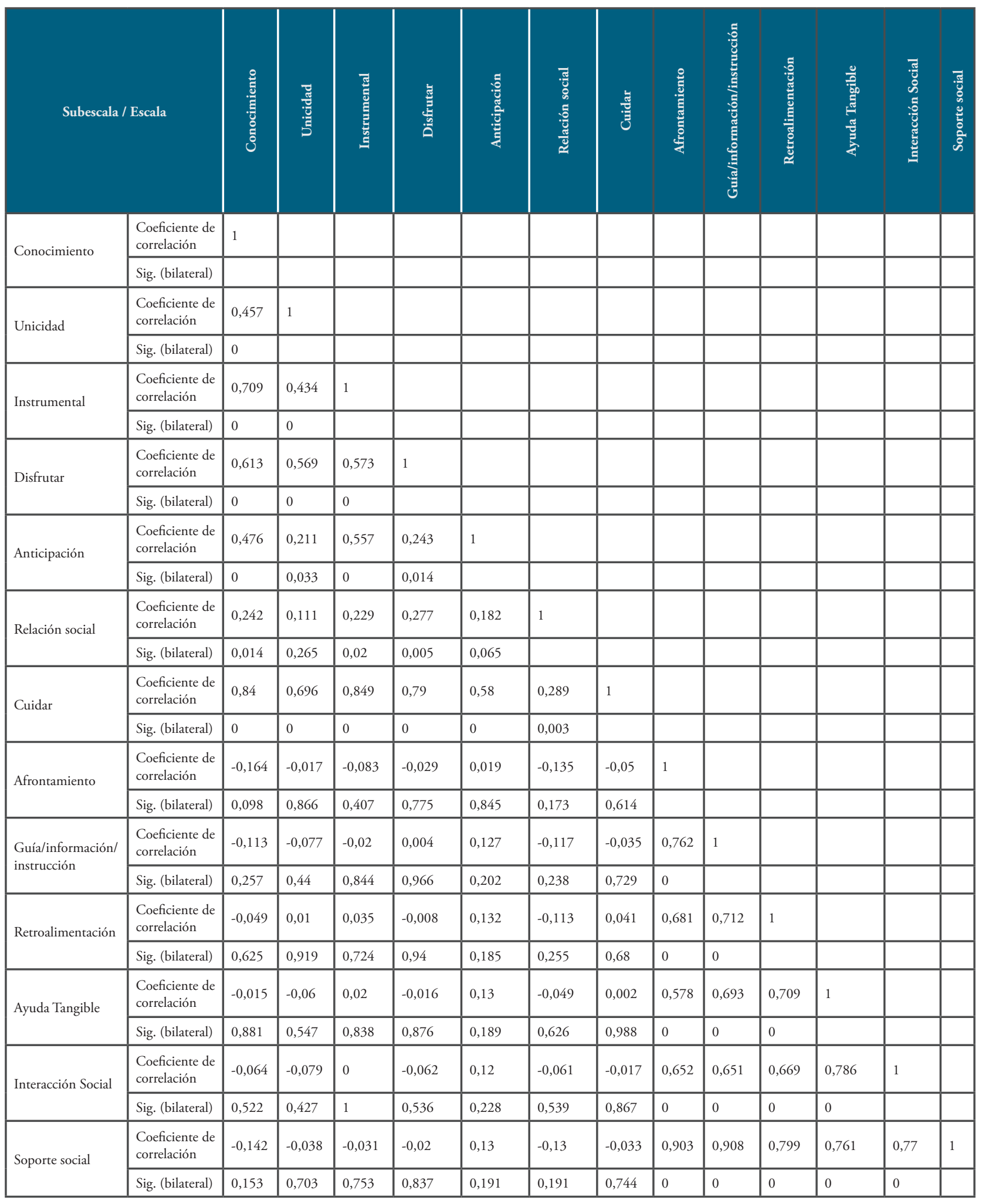

Fuente. Análisis resultados aplicación instrumentos. 


\section{Discusión}

En su mayoría, los cuidadores familiares de la provincia de Ocańa son mujeres, en edad productiva, con un nivel educativo medio y bajo, solteras y dedicadas al hogar o labores independientes. Características similares a las reportadas en la literatura (13-16). Históricamente, se reconoce el cuidado como algo innato a la naturaleza femenina y necesario para el mantenimiento de la vida y el desarrollo de la civilización (17). Sin embargo, en la región se encuentra un número significativo de hombres en el desempeño del rol de cuidador, lo cual puede explicarse desde los cambios sociales y demográficos como el aumento de las personas en condición de dependencia por edad o enfermedad, estructura familiar y cambio del rol de la mujer en la manutención del hogar, especialmente cuando son esposos o hijos (18). La experiencia de cuidar no ha sido muy abordada desde la perspectiva de género, específicamente en el contexto colombiano y latinoamericano, pero se documentan diferencias (19), que ameritan ser estudiadas a profundidad.

El nivel educativo se ha asociado a mejores conductas preventivas y al fortalecimiento de las relaciones personales, sociales e integración social frente a la enfermedad cróni$\mathrm{ca}$, lo que permite esperar mayor acceso y uso de información de apoyo para el desempeño del cuidado; así como los niveles educativos bajos y medios se asocian con mayor prevalencia de factores de riesgo. De otra parte, el contar con una relación de pareja es un factor de ayuda para muchos de los enfermos en la toma de decisiones y frente a la necesidad de ayuda para las actividades de la vida diaria cuando su salud se deteriora, con efectos en la salud y bienestar a largo plazo de quien asume la responsabilidad de cuidado. El cuidado de la enfermedad crónica se asocia a la ausencia de trabajo remunerado, lo cual disminuye la productividad y empobrece a las familias (20).

Respecto al tiempo en el desempeño de rol se encontró un promedio cercano a los 5 ańos, con cuidado desde el momento del diagnóstico en el $68 \%$ de los cuidadores con una dedicación entre 12 y 13 horas al día, aspectos en los que se encuentran resultados diversos en la literatura, en general, relacionados con la complejidad de la enfermedad crónica, el compromiso funcional, alteración cognitiva, tipo de enfermedad crónica de referencia para la realización del estudio $(12,13,21,22)$. Como lo señalan otras investigaciones sobre cuidadores con horas de dedicación al cuidado similares, el ejercicio del cuidado por tiempo prolongado puede tener efectos negativos en los cuidadores como sentimiento de carga, fatiga, mala salud, síntomas depresivos y afectación de la calidad de vida (23). El cuidado desde el diagnostico, el tiempo y horas dedicadas al cuidado del familiar enfermo, son elementos significativos de la construcción del vínculo y reconocimiento de aspectos intangibles de la relación y el cuidado (24).

En relación con la percepción de carga, los cuidadores del estudio reportan en un 95 
$\%$ algún nivel de carga, entre leve e intensa, resultado similar a lo reportado en otros estudios realizados en cuidadores familiares de personas con diferentes enfermedades crónicas como Alzheimer, Insuficiencia renal crónica y EPOC (25 -8). De manera general, estos resultados ponen en evidencia la situación que afrontan los cuidadores debido a los importantes cambios a nivel personal, familiar, social y laboral que trae a su vida el desempeño del rol, acompańados en ocasiones por problemas de salud y sentimientos de sobrecarga emocional y física.

Para la variable soporte social el estudio reportó que los cuidadores presentan una percepción alta de soporte social a nivel global y en las 5 subescalas que lo componen, con valores promedio iguales a 209.8 \pm 24.9 de los 228 puntos posibles. Estos resultados difieren de los hallazgos de otros estudios en el contexto colombiano, como el de Romero y colaboradores (7), quienes encontraron cuidadores familiares de personas con enfermedad crónica entre poco $y$ algo satisfechos con el soporte social percibido, con mayor afectación en la dimensión interacción social; y el Barrera Ortiz y colaboradores (16), quienes estudiaron el soporte social percibido en cuidadores y personas con enfermedad crónica de cinco macro regiones de Colombia, encontrando que los cuidadores de la región Andina, a la cual pertenece el municipio de Ocańa, presentaron niveles más bajos de soporte social percibido que la región de la Amazonía y Orinoquía.
Igualmente difieren de lo reportado por Puerto Pedraza (21), quien en su estudio con cuidadores familiares de personas en tratamiento para el cáncer encontró una percepción del soporte social media y baja, lo cual relaciona con la dimensión social - cultural del cuidado a las personas con enfermedades oncológicas debido a su asociación con el aislamiento, el dolor y la muerte.

La diferencia de los resultados obtenidos en este estudio en la variable soporte social indican la necesidad de adelantar estudios que exploren la asociación entre características sociodemográficas del cuidador, tipo y fase de la enfermedad crónica, así como aspectos culturales propios que puedan intervenir en la percepción de soporte social en la experiencia de la enfermedad crónica.

El soporte social es considerado un aspecto esencial en la interacción humana, un recurso para el afrontamiento, la adaptación y reducción del impacto de la enfermedad en pacientes y cuidadores aun en la diversidad y complejidad de la experiencia de la enfermedad crónica. Diferentes autores, destacan la importancia del soporte social en la enfermedad crónica para el funcionamiento social, la salud mental y física en la experiencia de cuidar (29-31).

En cuanto a la competencia para cuidar en el hogar en los cuidadores familiares de la provincia de Ocaña los resultados muestran que la mayoría tenían un nivel general alto, con igual tendencia en la totalidad 
de dimensiones que valora el instrumento. De manera general, esto evidencia que los cuidadores cuentan con la preparación, el conocimiento y las habilidades necesarias para dar cuidado a su familiar y responder a las necesidades, crear vínculo reconociendo sus limitaciones y afrontando las dificultades del desempeño del rol (32). Sin embargo, el nivel medio en las categorías conocimiento, instrumental y unicidad plantean la necesidad de acompańamiento y apoyo continuo a los cuidadores para el desarrollo de las competencias en el cuidado y el afrontamiento de los efectos del ejercicio del rol en la dimensión física, social, emocional, laboral, familiar y económica. Algunas condiciones que pueden haber favorecido la competencia para cuidar son el vínculo entre la diada, el tiempo en el desempeño como cuidador y el cuidado desde el diagnóstico, aunque no se estableció relación mediante pruebas estadísticas. Podría inferirse que la competencia es un aspecto que evoluciona con el tiempo y la práctica.

Estos resultados difieren de los hallazgos de otros estudios a nivel nacional que evidencian que los cuidadores no cuentan con niveles óptimos para desarrollar el rol que asumen $(6,8,15,22,31,32)$, ya que allí encontraron niveles de competencia medio y bajos en cuidadores de personas con diferentes condiciones crónicas, lo cual no permite afirmar que los niveles de competencia estén relacionados con la complejidad de la situación y nivel de dependencia del receptor de cuidado.
No se identificó relación entre el soporte social y la competencia para cuidar, las dos variables se comportan de manera independiente, aun cuando ambas obtuvieron niveles satisfactorios y altos respectivamente. Es innegable la importancia de estos elementos en el cuidado de la enfermedad crónica, representan un apoyo para la persona que enfrenta la condición y para el cuidador que acompańa el proceso, influyendo de manera directa en los resultados alcanzados y bienestar de la persona en condición de cronicidad, la calidad del cuidado, la disminución del impacto de la enfermedad en la familia y en la carga en el cuidador.

Cuidadores familiares con una adecuada competencia representan un factor positivo para el control de la enfermedad crónica, favoreciendo los procesos de adaptación a los cambios y nuevos requerimientos para el paciente relacionados con la enfermedad. El desarrollo de programas e intervenciones por parte enfermería dirigidas a fortalecer el desarrollo de las capacidades, conocimientos, habilidades y actitudes en los cuidadores familiares deben ser continuas y acompañar el proceso de manera permanente para responder a las necesidades no solo del paciente sino del cuidador, fortalecer la autogestión y el autocuidado, para garantizar un cuidado de calidad y seguro, y evitar de ser posible la sobrecarga del cuidador en la experiencia de acompañar a su ser querido en esa etapa de la vida(33-36). 


\section{Conclusiones}

El nivel de competencia para el cuidado en el hogar en los cuidadores de Ocańa puede calificarse de adecuado a nivel global y por dimensiones. Sin embargo, se identifican necesidades de fortalecimiento en el conocimiento, lo instrumental y unicidad. Se evidencia relación directa entre el género femenino y la mayor competencia para el cuidado, lo cual pone en evidencia la vulnerabilidad de la mujer cuidadora en razón a los múltiples roles que desempeña.

Respecto al soporte social los resultados evidencian que los cuidadores se encuentran satisfechos en todas las dimensiones de la escala de Hilbert, especialmente en interacción social y ayuda tangible, identificándose relación estadísticamente significativa entre el mayor nivel de escolaridad y la mayor satisfacción global con el soporte social percibido.

Los resultados del estudio evidencian que la competencia para cuidar en el hogar en el cuidador se desarrolla de manera independiente a la satisfacción con el soporte social percibido.

\section{Referencias}

1. Landínez N. Manual del cuidador de personas mayores. Bogotá: Universidad Nacional de Colombia; 2016.

2. Naciones Unidas. Envejecimiento. [Internet]. [citado 10 Ene 2018]; Disponible en: http://www. un.org/es/sections/issues-depth/ageing/index.html
3. Correa L, Uribe L. La vejez de hoy y del mañana en Colombia. Bogotá: Fundación Saldarriaga Concha; 2017.

4. Flórez C, Villar L, Puerta N, Berrocal L. Proceso de Envejecimiento de la Población en Colombia. En Misión Colombia Envejece: Cifras, retos y recomendaciones. Bogotá: Fundación Saldarriaga Concha publicaciones; 2015.

5. Beltrán J, Morcillo R, Rivas A. El cuidado familiar y vocacional en la persona mayor. Bogota: Colegio Mayor de Cundinamarca; 2014

6. Vega O, Peñaranda E, Rico Y, Rozo Y. Family caregivers: Competence in the care of the chronically ill. Rev. cienc. cuidad. 2018; 15(1):7-17.

7. Romero S, Correa B, Camargo F. Soporte social percibido en cuidadores familiares de personas con enfermedad crónica. Rev Univ. Salud. 2017; 19(1):8593.

8. Carrillo M, Barreto R, Arboleda L, Gutiérrez O, Melo B, Támara V. Competencia para cuidar en el hogar de personas con enfermedad crónica y sus cuidadores en Colombia: 2015. Fac. Med. 2015; 63(4): 665-75

9. Herrera A, Flórez I, Romero E, Montalvo A. Soporte social a cuidadores familiares de personas con enfermedad crónica en Cartagena. Aquichán 2012; 12(3):286-97.

10. Hilbert GA. Social support in chronic illness. In: Strickland O, Walltz C, Wheeler K, editors. Measurement of nursing outcomes: measuring client self-care and coping skills. New York: Springer; 1990: 79-95.

11. Ministerio de Salud de Colombia. Resolución No 008430 de 1993. Por lo cual se establecen las normas científicas, técnicas y administrativas para la investigación en salud. Bogotá; Congreso de la Republica; 1993.

12. Carrillo GM, Sánchez Herrera B, Arias Rojas EM. Validation of an instrument to assess the homecare competency of the family caregiver of a person with 
chronic disease. Invest. Educ. Enferm. [Internet] 2015 [Consultado, 25 Abril 2018]; 33(3):449-455. Disponible en: http://doi.org/87m

13. Arias M, Barrera L, Carrillo G, Chaparro L, Sánchez B, Vargas E. Cuidadores familiares de personas con enfermedad crónica en las regiones de frontera colombiana: perfil y carga percibida de cuidado. Revista de la Facultad de Medicina [Internet] 2014 [Consultado 17 abril de 2019]; 62(3):387-397. Disponible en: doi:https://doi.org/10.15446/revfacmed.v62n3.39091.

14. Achury DM, Restrepo A, Torres NM, Buitrago AL, Neira NX, Devia P. Competencia de los cuidadores familiares para cuidar a los pacientes con falla cardíaca. Rev Cuid. 2017; 8(3): 1721-32.

15. Cantillo-Medina CP, Ramírez-Perdomo CA, Perdomo-Romero AY. Habilidad de cuidado en cuidadores familiares de personas con enfermedad crónica y sobrecarga percibida. Cienc. enferm. [Internet]. 2018 [citado 2019 Abr 17]; 24: 16. Disponible en: http://dx.doi.org/10.4067/s071795532018000100216.

16. Barrera-Ortiz L, Campos MS, Gallardo-Solarte K, Coral-Ibarra RC, Hernández-Bustos A. Soporte social percibido por las personas con enfermedad crónica y sus cuidadores familiares en cinco macro regiones geográficas de Colombia. Rev Univ. salud. 2016;18(1):102112

17. Fernández-Tijero MC. El origen de la mujer cuidadora: apuntes para el análisis hermenéutico de los primeros testimonios. Index Enferm [Internet]. 2016 [citado 2019 Abr 17]; 25(1-2): 93-97. Disponible en: http://scielo.isciii.es/scielo.php?script=sci_ arttext\&pid=S1132-12962016000100021\&lng=es.

18. Sanchez B, Carrillo M, Corredor L. Caracterización y carga del cuidado en hombres cuidadores familiares de personas con enfermedad crónica en Colombia. Revista Archivos de Medicina [internet] 2014. [Consultado 17 de abril 2019] 14(2):219230. Disponible en: http://revistasum.umanizales. edu.co/ojs/index.php/archivosmedicina/article/ view/299/783

19. Barrera L, Pinto N, Sánchez B. Habilidad de cuidado de cuidadores familiares de personas con enfermedad crónica: comparación de géneros. Actualizaciones en Enfermería [Internet] 2006 [consultado 17 de abril de 2019]; 9(2):9-13. Disponible en: https:/encolombia.com/medicina/revistas-medicas/ enfermeria/ve-92/enfermeria9206-habilidad/

20. Chaparro L, Sánchez B, Carrillo GM. Encuesta de caracterización del cuidado de la diada cuidador familiar - persona con enfermedad crónica. Rev. cien. cuidad. 2014; 11(2): 31-45.

21. Puerto-Pedraza HM, Carrillo-González GM. Calidad de vida y soporte social en los cuidadores familiares de personas en tratamiento contra el cancer. Rev Univ Ind Santander Salud. 2015; 47(2): 125136.

22. Torres-Pinto X, Carreño-Moreno S, Chaparro-Díaz L. Factores que influencian la habilidad y sobrecarga del cuidador familiar del enfermo crónico. Rev Univ Ind Santander Salud. 2017; 49(2): 330-338.

23. Abellan, M. J., López, C., Santi, M. J., Deudero, M. y Picardo, J. M. (2016). ¿Quién es el receptor de intervenciones sociosanitarias y cuáles son sus necesidades? Enfermería Clínica [Internet] 2016[ consultado 10 de septiembre de 2019]; 26(1): 49-54. doi: 10.1016/j.enfcli.2015.08.004. Disponible en: https://www.elsevier.es/es-revista-enfermeria-clinica-35-articulo-quien-es-el-receptor-intervenciones-S1130862115001151

24. Chaparro L. El vínculo especial de cuidado: construcción de una teoría fundamentada. Avances en enfermería. 2010; 28(2):123-133.

25. Arechabala MC, Catoni MI, Palma E, Barrios S. Depresión y autopercepción de la carga del cuidado en pacientes en hemodiálisis y sus cuidadores. Rev Panam Salud Publica. 2011;30(1):74-9.

26. Pinzón EA, Carrillo GM. Carga del cuidado y calidad de vida en cuidadores familiares de personas con 
enfermedad respiratoria crónica. Rev. Fac. Nac. Salud Pública 2016; 34(2): 193-201. DOI: 10.17533/ udea.rfnsp.v34n2a08

27. Pérez-Perdomo M, Cartaya-Poey M, Olano-Montes BL. Variables asociadas con la depresión y la sobrecarga que experimentan los cuidadores principales de los ancianos con Alzheimer. Rev Cubana Enfermer [Internet]. 2012 [citado 2019 Abr 17]; 28(4): 509-520. Disponible en: http:// scielo.sld.cu/scielo.php?script=sci_arttext \&pi$\mathrm{d}=$ S0864-03192012000400007\&lng=es.

28. Romero-Massa E, Bohórquez-Moreno C, Castro-Muñoz K. Calidad de vida y sobrecarga percibida por cuidadores familiares de pacientes con enfermedad renal crónica, Cartagena (Colombia). Arch Med [Internet] 2018; [Consultato 17 abril 2019]: 18(1):105-3. Disponible en: DOI: https:// doi.org/10.30554/archmed.18.1.2520.2018.

29. Puerto HM. Soporte social percibido en cuidadores familiares de personas en tratamiento contra el cáncer. Rev Cuid. 2017; 8(1): 1407-22. http://dx.doi. org/10.15649/cuidarte.v8i1.345

30. Gómez M, Lagoueyte M. El apoyo social: estrategia para afrontar el cáncer de cérvix. Avances en Enfermería [Internet] 2012 [Consultado 17 abril 2019]; 30(1):32-41. Disponible en: https:// revistas.unal.edu.co/index.php/avenferm/article/ view/35430/36127

31. Godoy AM, Pérez MA, Doménech del Rio A, Prunera MJ. Caregiver burden and social support perceived by patients with chronic obstructive pulmonar disease. Rev Calid Asist. [Internet] 2014; [Consultado 17 abril 2019]; 29(6):320-324. Disponible en: https://www.sciencedirect.com/science/article/pii/ S1134282X14001171?via\%3Dihub\#abst0020

32. Carrillo González GM, Sánchez Herrara B, Vargas Rosero E. Desarrollo y pruebas pscométricas del Instrument "cuidar" - versión corta para medir la competencia del cuidado en el hogar. Rev Univ Ind San- tander Salud. 2016; 48(2): 222-231. DOI: http:// dx.doi.org/10.18273/revsal.v48n2-2016007

33. Sánchez LM, Carrillo GM. Competencia para el cuidado en el hogar diada persona con cáncer en quimioterapia-cuidador familiar Psicooncología 2017;14: 137-48. DOI: 10.5209/PSIC.55817

34. Aldana E, Barrera S, Rodríguez K, Gómez, O, Carrillo G. Competencia para el cuidado (CUIDAR) en el hogar de personas con enfermedad renal crónica en hemodiálisis. Enfermer Nefrol. 2016; 19(3): 265-73.

35. Carreño Moreno, S., Chaparro Díaz, L., Criado, L., Vega, O., \& Cuenca, I. (2018). Magnitud de efecto de un programa dirigido a cuidadores familiares de personas con enfermedad crónica. NOVA, 16(29), 11-20. https://doi.org/10.22490/24629448.2684

36. Panche Castellanos, K. C., Rangel Acosta, L., \& Corrales Ramírez, L. C. (2013). Uso de dispositivos de micronebulización en adultos mayores en un programa de hospitalización domiciliaria. NOVA, 11(20). https://doi.org/10.22490/24629448.1034 